Research Article

\title{
Behavior of a Free Dual-Spin Gyrostat with Different Ratios of Inertia Moments
}

\author{
Vladimir Aslanov \\ Department of Theoretical Mechanics, Samara State Aerospace University, 34 Moskovskoe Shosse, Samara 443086, Russia
}

Correspondence should be addressed to Vladimir Aslanov; aslanov_vs@mail.ru

Received 12 October 2014; Accepted 27 December 2014

Academic Editor: Alkesh Punjabi

Copyright (C) 2015 Vladimir Aslanov. This is an open access article distributed under the Creative Commons Attribution License, which permits unrestricted use, distribution, and reproduction in any medium, provided the original work is properly cited.

\begin{abstract}
The attitude motion is studied of asymmetric dual-spin gyrostats which may be modeled as free systems of two rigid bodies, one asymmetric and one axisymmetric. Exact analytical solutions of the attitude motion are presented for all possible ratios of inertia moments of these bodies. The dynamics of free gyrostats with zero internal torque is considered. The dimensionless nonlinear equations of the gyrostat are written in Serret-Andoyer canonical variables. The previously known exact solutions are complemented by new several solutions in terms of Jacobi elliptic functions. The results of the study can be useful for the analysis of dual-spin spacecraft dynamics.
\end{abstract}

\section{Introduction}

The dynamics of rotating bodies is a classic topic of study in mechanics. In the eighteenth and nineteenth centuries, several aspects of the motion of a rotating rigid body were studied by such famous mathematicians as Euler, Cauchy, Jacobi, Poinsot, Lagrange, and Kovalevskaya. However, the study of the dynamics of rotating bodies is still very important for numerous applications such as the dynamics of satellite gyrostat, spacecraft, robotics, and the like. In this paper we deal with a particular case of gyrostat; it consists of a platform with a triaxial ellipsoid of inertia and a symmetrical rotor whose axis of symmetry is aligned along one of the principal axes of inertia of the platform.

The paper deals with the analytical solutions of the attitude motion of the axial gyrostats. The analytical solutions of determining the attitude motion of the axial gyrostat with a free rotor (see, e.g., [1]) are relatively straightforward. Furthermore, Kane [2] has given the solution for the attitude motion of an axial gyrostat with a driven rotor. In many papers (see, e.g., [3-16]), this modern problem is usually performed by following two distinct steps. The dynamics of the rotation are represented by using differential equations written for the components of the angular momentum, and then the kinematic equations are utilized to transform the angular momentum. While this classical formulation is widespread, scientists also exhibit a preference for the Serret-Andoyer variables (SA) variables, which allow performing convenient analysis of the attitude motion of the gyrostats. Serret [17] discovered these variables by solving the Hamilton-Jacobi equation written in terms of Eulerian coordinates. Andoyer [18] used spherical trigonometry to show that the Serret transformation was simply a change of Eulerian coordinates that depended upon the angular momentum components. Deprit [19] and Deprit and Elipe [20] established the canonicity of the Serret transformation by using differential forms and without resorting to finding a generating function. SA variables are used also in Hamiltonian structure of an asymmetric gyrostat in the gravitational field [21]. The SA variables are used to describe the Hamiltonian equations for attitude motions of the axial free gyrostat [22-26]. Euler angles and angular velocities of the gyrostat as functions of the SA variables are given in Aslanov [27].

Hall [6] proposed a procedure based upon the global analysis of the rotational dynamics. Hall and Rand [7] considered spinup dynamics of classical axial gyrostat composed of an asymmetric platform and an axisymmetric rotor. They obtained the averaged equations for slowly varying relative rotation of the bodies and analytical solutions for the projections of angular momentum in the case of constant relative rotation. Cochran et al. [3] have obtained analytical 
solutions of the attitude motion of the free gyrostats. Elipe [8] investigated a free gyrostat with three flywheels rotating about the three principal axes of inertia and without any external forces or torques. Elipe and Lanchares [28] have introduced coordinates to represent the orbits of constant angular momentum as a flow on a sphere. This representation has shown that the problem is equivalent to a quadratic Hamiltonian depending on two parameters.

This paper focuses on the study of the attitude motion of the gyrostat $P+R$ consisting of an axisymmetric rotor $R\left(A_{r}=\right.$ $\left.B_{r}, C_{r}\right)$ and of an unbalanced platform $P\left(A_{p}>B_{p}, C_{p}\right)$. This paper is a development of Aslanov's works [23, 24, 27] in which we studied three types of the gyrostats: oblate $\left(C_{p}>\right.$ $\left.A_{p}+A_{r}>B_{p}+A_{r}\right)$, intermediate $\left(A_{p}+A_{r}>C_{p}>B_{p}+A_{r}\right)$, and prolate $\left(A_{p}+A_{r}>B_{p}+A_{r}>C_{p}\right)$. Here we add two new types of the gyrostats: oblate-intermediate $\left(C_{p}=A_{p}+A_{r}>\right.$ $\left.B_{p}+A_{r}\right)$ and prolate-intermediate $\left(A_{p}+A_{r}>C_{p}=B_{p}+A_{r}\right)$, and we do not impose restrictions on the angular velocity of rotation of the rotor relative to the platform as in the papers $[23,24]$.

The paper is divided into five sections. In Section 2 the dimensionless differential equations of the motion variables and the stationary solutions are presented in terms of the SA variables. In Section 3 we find singular points on a phase plane for various types of the gyrostats. Section 4 focuses on an integration of the motion equations in terms of Jacobi elliptic functions. Section 5 presents a complete set of the phase trajectories and the exact solutions.

\section{Canonical Equations and Stationary Solutions}

Let us consider a motion of a gyrostat in the absence of external torque and internal torque applied by $P$ on $R$ about axis $O z$. We set the platform's frame Oxyz relative to an inertial frame $O X Y Z$, using the canonical SA variables [24]: $l, g, \widetilde{h}, L, G, \widetilde{H}$. The orientation of the rotor relative to the platform's frame $O x y z$ is defined by the angle $\delta$ (Figure 1). The Hamiltonian for the torque-free motion with use of the SA variables and pair of variables $\left(\delta, p_{\delta}\right)$ is [22]

$$
\begin{aligned}
H_{0} & =\frac{G^{2}-L^{2}}{2}\left[\frac{\cos ^{2} l}{A_{r}+B_{p}}+\frac{\sin ^{2} l}{A_{r}+A_{p}}\right]+\frac{\left(L-p_{\delta}\right)^{2}}{2 C_{p}}+\frac{p_{\delta}^{2}}{2 C_{r}} \\
& =h_{0}=\text { const, }
\end{aligned}
$$

where $p_{\delta}=\partial T / \partial \dot{\delta}$ is the generalized momentum and $T$ is the kinetic energy of the gyrostat. Since $g, \widetilde{h}$, and $\delta$ are cyclic and $\widetilde{H}$ does not appear in the Hamiltonian, thus $G, \widetilde{h}, \widetilde{H}$, and $p_{\delta}$ are constants, which means that the angular momentum vector is conserved and the first integrals of the motion are

$$
\begin{gathered}
G=\sqrt{h_{x}^{2}+h_{y}^{2}+h_{z}^{2}}=\text { const }, \\
p_{\delta}=\text { const },
\end{gathered}
$$

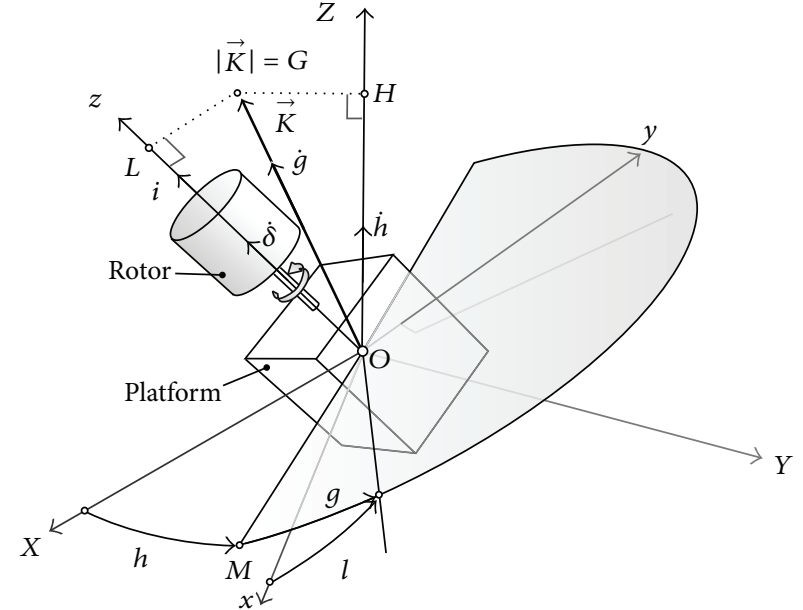

FIgURE 1: The axial gyrostat.

where components of the angular momentum vector in the coordinate system $O x y z$ are

$$
\begin{gathered}
h_{x}=\sqrt{G^{2}-L^{2}} \sin l, \quad h_{y}=\sqrt{G^{2}-L^{2}} \cos l, \\
h_{z}=L .
\end{gathered}
$$

Since the number of degrees of freedom of the torque-free motion of the gyrostat is reduced to one, the motion

$$
\dot{l}=\frac{\partial H_{0}}{\partial L}, \quad \dot{L}=-\frac{\partial H_{0}}{\partial l} .
$$

The transformation of (4) to a dimensionless form is obtained by scaling the two momentums and time, as follows:

$$
s=\frac{L}{G}, \quad d=\frac{p_{\delta}}{G}=\frac{C_{r}\left(L+C_{p} \dot{\delta}\right)}{\left(C_{p}+C_{r}\right) G}, \quad \tau=\frac{G}{C_{p}} t .
$$

Derivatives with respect to $\tau$ are denoted by a derivative sign: $x^{\prime}=d x / d \tau$. The change of variables (5) leads to the to the equivalent set of dimensionless equations:

$$
\begin{gathered}
l^{\prime}=\frac{\partial H}{\partial s}=s-d-\frac{1}{2} s(a+b+(b-a) \cos 2 l), \\
s^{\prime}=-\frac{\partial H}{\partial l}=\frac{1}{2}(b-a)\left(1-s^{2}\right) \sin 2 l .
\end{gathered}
$$

Here $a=C_{p} / A$ and $b=C_{p} / B$ are the relative reciprocals of the principal moments of inertia of the gyrostat $A=A_{p}+A_{r}$ and $B=B_{p}+A_{r}$, respectively. $H$ is Hamiltonian by [24]

$$
\begin{aligned}
H & =\frac{1}{4}[a+b+(b-a) \cos 2 l]\left(1-s^{2}\right)+\frac{1}{2} s^{2}-s d \\
& =h=\text { const. }
\end{aligned}
$$

Solving expression (8) with respect to $\cos 2 l$, we obtain the phase trajectories:

$$
\cos 2 l=\frac{(a+b-2) s^{2}+4 d s+4 h-a-b}{\left(1-s^{2}\right)(b-a)} .
$$




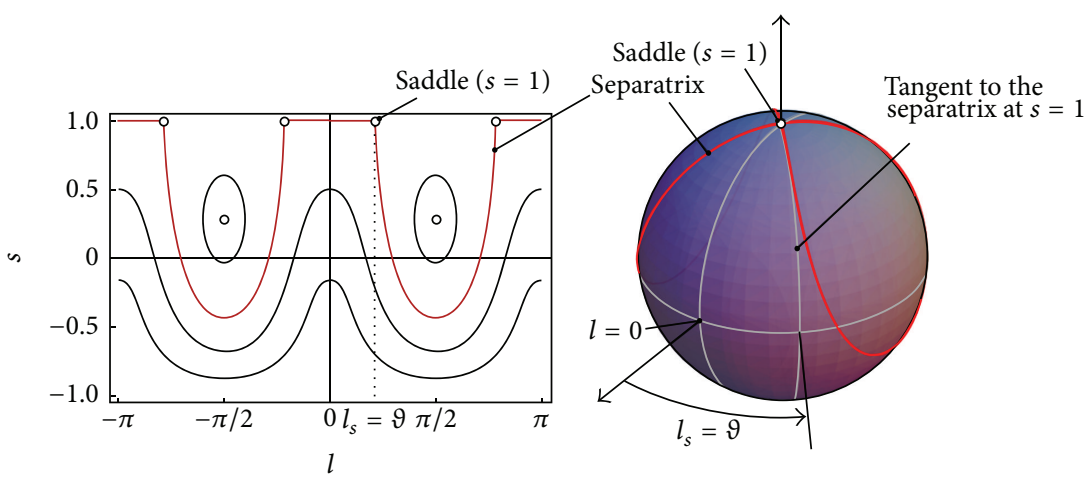

FIGURE 2: Separatrices in the plane and on the unit sphere.

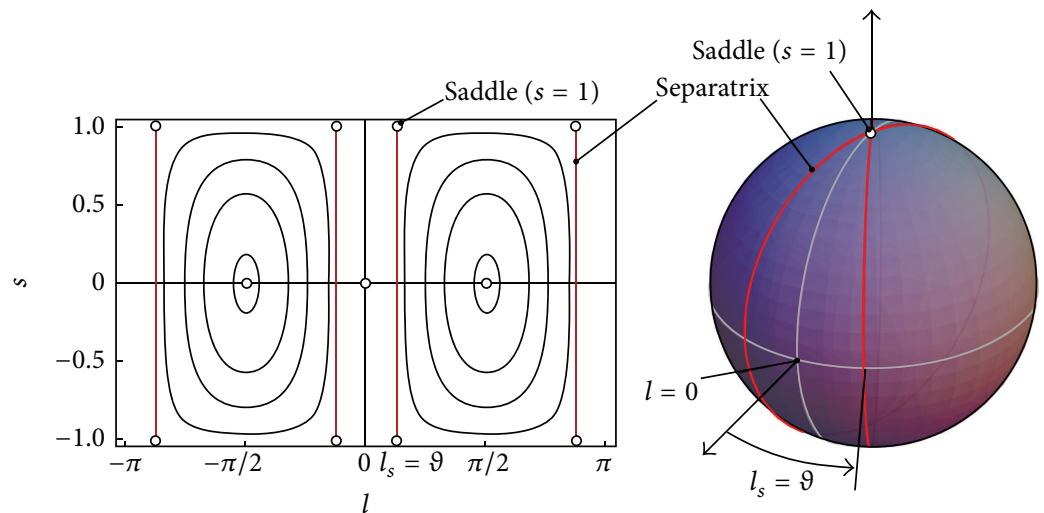

Figure 3: Separatrices in the plane and on the unit sphere for Euler's case.

By equating to zero (6) and (7), we get

$$
\begin{gathered}
2(s-d)-s(a+b+(b-a) \cos 2 l)=0, \\
(b-a)\left(1-s^{2}\right) \sin 2 l=0 .
\end{gathered}
$$

Solving (10), we have four stationary solutions [24]:

$$
\begin{gathered}
\cos \left(2 l_{*}\right)=1, \quad s_{*}=\frac{d}{(1-b)}, \\
\cos \left(2 l_{*}\right)=-1, \quad s_{*}=\frac{d}{(1-a)}, \\
\cos \left(2 l_{*}\right)=\frac{(2-a-b-2 d)}{(b-a)}, \quad s_{*}=1, \\
\cos \left(2 l_{*}\right)=\frac{(2-a-b+2 d)}{(b-a)}, \quad s_{*}=-1 .
\end{gathered}
$$

The first stationary solution (11) is stable if $b>1\left(C_{P}>B\right)$ and is unstable if $b<1\left(C_{P}<B\right)$. The second stationary solution (12) is stable if $a<1\left(C_{P}<A\right)$ and unstable if $a>1\left(C_{P}>\right.$ $A$ ). The third and fourth stationary solutions (13) and (14) are always unstable.

Figure 2 shows phase space on plane and unit sphere for solutions (13). An angle between the tangents to the separatrix and to the line $\vartheta=0$ in the vicinity $s_{*}= \pm 1$ is equal to the angle $\vartheta=l_{*}$. Next we will study the trajectories in the phase space, except the separatrices which contain singular points (11)-(14).

It is obvious that the roots of (13) and (14) give ambiguous solutions when $d=0$ (Euler's case, Figure 3 ), so this particular case is excluded from consideration. The wellknown solutions of Euler's Equations of motion of a rigid body are studied in terms of the SA variables in the papers $[29,30]$.

Other authors $[3,7,9]$ formulated the problem in terms of the angular momentum components (3):

$$
\begin{gathered}
h_{x}=G \sqrt{1-s^{2}} \sin l, \quad h_{y}=G \sqrt{1-s^{2}} \cos l, \\
h_{z}=G s,
\end{gathered}
$$

which avoid the singularity in the points (13) and (14)

$$
h_{x *}=0, \quad h_{y *}=0, \quad h_{z *}= \pm G .
$$

The Hamiltonian (8) has the form for the axisymmetric platform $A_{p}=B_{p} \Rightarrow(a=b)$ :

$$
H(s)=\frac{a\left(1-s^{2}\right)}{2}+\frac{s^{2}}{2}-s d=h=\text { const. }
$$

In this case the attitude motion is a precession around the vector of the angular momentum $\vec{K}$ with a constant angle $\alpha=\arccos (s)$. 
TABle 1: The singular points.

\begin{tabular}{|c|c|c|c|c|c|c|}
\hline \multirow{3}{*}{ Gyrostat type } & \multirow{3}{*}{ Subtype } & \multirow{3}{*}{ Kinematical conditions } & \multicolumn{4}{|c|}{ The singular points $(l, s)$} \\
\hline & & & \multicolumn{2}{|c|}{ Centers } & \multicolumn{2}{|c|}{ Saddles } \\
\hline & & & $l_{c}$ & $s_{c}$ & $l_{s}$ & $s_{s}$ \\
\hline \multirow{4}{*}{$\begin{array}{l}\text { Oblate } \\
C_{P}>A>B \\
(b>a>1)\end{array}$} & $\mathrm{Oa}$ & $V_{a} \leq 1$ & 0 & $d$ & $+\frac{\pi}{2}$ & $d$ \\
\hline & & & & $1-b$ & $\frac{1}{2}$ & $1-a$ \\
\hline & $O \mathrm{~b}_{\mathrm{s}}$ & $V>1$ & 0 & $\frac{a}{1-b}$ & & \\
\hline & 00 & $v_{a}>1$ & $+\frac{\pi}{-}$ & $d$ & $y^{+}$ & $-\sin d$ \\
\hline \multirow{3}{*}{$\begin{array}{l}\text { Oblate-intermediate } \\
C_{P}=A>B \\
(b>a=1)\end{array}$} & & & $\pm \frac{1}{2}$ & $\overline{1-a}$ & $l_{s}$ & $-\sin a$ \\
\hline & Oi & $V_{a} \rightarrow \infty$ & 0 & $d$ & & \\
\hline & Ia & $V_{a} \geq 1$ & & $1-b$ & & \\
\hline \multirow{2}{*}{$\begin{array}{l}\text { Intermediate } \\
A>C_{P}>B \\
(b>1>a)\end{array}$} & $\mathrm{Ib}$ & $V_{a}<1, V_{b}<1$ & 0 & $\frac{d}{1-b}$ & $l_{s}^{+}$ & \\
\hline & & & $\pm \frac{\pi}{2}$ & $\frac{d}{1-a}$ & $l_{s}^{-}$ & $\sin d$ \\
\hline \multirow[b]{2}{*}{$\begin{array}{l}\text { Prolate-intermediate } \\
A>C_{P}=B \\
(b=1>a)\end{array}$} & Ic & $V_{b} \geq 1$ & $\pi$ & $d$ & & \\
\hline & $\mathrm{Pi}$ & $V_{b} \rightarrow \infty$ & $\pm \overline{2}$ & $\overline{1-a}$ & & \\
\hline \multirow{3}{*}{$\begin{array}{l}\text { Prolate } \\
A>B>C_{P} \\
(1>b>a)\end{array}$} & $\mathrm{Pa}$ & $V_{b}>1$ & 0 & $\frac{d}{1-b}$ & $l_{s}^{-}$ & $\sin d$ \\
\hline & & & $\pm \frac{\pi}{2}$ & $\frac{d}{1-a}$ & & \\
\hline & $\mathrm{Pb}$ & $V_{b} \leq 1$ & $\pm \frac{\pi}{2}$ & $\frac{d}{1-a}$ & 0 & $\frac{d}{1-b}$ \\
\hline
\end{tabular}

\section{Types of the Gyrostats and Singular Points in the Phase Space}

The five basic types of the gyrostats are determined by the following ratios of the inertia moments:

(1) oblate gyrostat: $C_{P}>A>B(b>a>1)$,

(2) oblate-intermediate gyrostat: $C_{P}=A>B(b>a=$ 1),

(3) intermediate gyrostat: $A>C_{P}>B(b>1>a)$,

(4) prolate-intermediate gyrostat: $A>C_{P}=B(b=1>$ a),

(5) prolate gyrostat: $A>B>C_{P}(1>b>a)$.

The singular points corresponding to stationary solutions (11)-(14) for all types of gyrostats are shown in Table 1 . The table notations are as follows:

$$
V_{\gamma}=\left|\frac{d}{1-\gamma}\right| \quad(\gamma=a, b), \quad \cos 2 l_{s}^{ \pm}=\frac{2-a-b \pm 2 d}{b-a}
$$

\section{The Exact Solutions}

4.1. Integration of the Canonical Equations by Quadrature. In this formulation the exact solutions of (6) and (7) are presented only for the three subtypes [24]: $\mathrm{Oa}, \mathrm{Ib}$, and $\mathrm{Pb}$. To find all possible kinds of the solutions we use (7) and (9):

$$
\begin{gathered}
\tau= \pm \int \frac{d s}{\sqrt{F(s)}}+\text { const, } \\
F(s)=-4 f_{a}(s) f_{b}(s), \\
f_{\gamma}(s)=\frac{1}{2}(1-\gamma) s^{2}-d s+\frac{\gamma}{2}-h, \quad \gamma=a, b .
\end{gathered}
$$

Function (20) is a polynomial of the third degree for the two subtypes $\mathrm{Oi}(a=1)$ or $\mathrm{Pi}(b=1)$ :

$$
\begin{aligned}
F_{3}(s) & =\left(s-s_{1}\right)\left(s-s_{2}\right)\left(s-s_{3}\right) \\
& =a_{1} s^{3}+a_{2} s^{2}+a_{3} s+a_{4} .
\end{aligned}
$$

For all other subtypes function (20) is a polynomial of degree four:

$$
\begin{aligned}
F_{4}(s) & =\left(s-s_{1}\right)\left(s-s_{2}\right)\left(s-s_{3}\right)\left(s-s_{4}\right) \\
& =a_{0} s^{4}+a_{1} s^{3}+a_{2} s^{2}+a_{3} s+a_{4} .
\end{aligned}
$$

Real roots are numbered as follows:

$$
s_{4}<s_{3}<s_{2}<s_{1} \text {. }
$$


Complex conjugate roots are written as

$$
s_{m, n}=s_{s} \pm i s_{k}
$$

Let us denote the roots of the degree four (20):

$$
\begin{gathered}
s_{1,2}^{\gamma}(h)=\frac{\left(d \pm \sqrt{D_{\gamma}(h)}\right)}{(1-\gamma)}, \\
D_{\gamma}(h)=d^{2}+(2 h-\gamma)(1-\gamma), \quad \gamma=a, b .
\end{gathered}
$$

4.2. Various Types of the Exact Solutions. Case $\left(F(s)=F_{4}(s)\right.$, $\left.a_{0}<0\right)$. The integral (19) can be written as

$$
\lambda_{(-)} \tau=\int_{s_{2}}^{s} \frac{d s}{\sqrt{\left(s_{1}-s\right)\left(s-s_{2}\right)\left(s-s_{3}\right)\left(s-s_{4}\right)}},
$$

where $\lambda_{(-)}=\sqrt{\left(A-C_{p}\right)\left(B-C_{p}\right) /(A B)}$.

Two Real Roots and Two Complex Conjugate Roots. The roots (25) include two real roots $s_{1}>s_{2}$ and two complex conjugate roots $s_{3,4}=s_{s} \pm i s_{k}$. Change of variable [31]

$$
\left(\tan \frac{\varphi}{2}\right)^{2}=\frac{\cos \theta_{1}}{\cos \theta_{2}} \frac{s_{1}-s}{s-s_{2}}=\frac{1-\cos \varphi}{1+\cos \varphi}
$$

converts the integral (26) to the Legendre normal form

$$
\omega \tau=\int_{\pi}^{\varphi} \frac{d \varphi}{\sqrt{1-k^{2} \sin ^{2} \varphi}}
$$

where

$$
\begin{gathered}
\omega=-\frac{\lambda_{(-)} s_{k}}{\sqrt{\cos \theta_{1} \cos \theta_{2}}}, \quad k=\sin \frac{\theta_{1}-\theta_{2}}{2}, \\
\tan \theta_{1}=\frac{s_{1}-s_{s}}{s_{k}}, \quad \tan \theta_{2}=\frac{s_{2}-s_{s}}{s_{k}}
\end{gathered}
$$

$\left(\theta_{1}, \theta_{2}\right.$ are acute angles $)$.

Taking into account (27) and (28), we get

$$
s=\frac{\left(c_{1} s_{1}+c_{2} s_{2}\right)+\left(c_{2} s_{2}-c_{1} s_{1}\right) c n\left[\omega\left(\tau-\tau_{0}\right), k\right]}{\left(c_{1}+c_{2}\right)+\left(c_{2}-c_{1}\right) c n\left[\omega\left(\tau-\tau_{0}\right), k\right]},
$$

where $c_{1}=\cos \theta_{1}, c_{2}=\cos \theta_{2}$, and $\operatorname{cn}[u, k]$ is an elliptic cosine. The arbitrary constant $\tau_{0}$ is equal to zero if $s_{0}=s_{2}$.

Four Real Roots. If the roots (25) are real and they correspond to the motion: $-1 \leq s_{4} \leq s \leq s_{3}$ and $s_{2} \leq s \leq s_{1} \leq 1$, then the exact solutions can be written as

$$
s=\frac{s_{2} s_{31}-s_{3} s_{21} \operatorname{sn}^{2}\left[\omega\left(\tau-\tau_{0}\right), k\right]}{s_{31}-s_{21} \operatorname{sn}^{2}\left[\omega\left(\tau-\tau_{0}\right), k\right]} \quad \text { if } s_{2} \leq s \leq s_{1},
$$

$$
s=\frac{s_{4} s_{31}+s_{1} s_{43} \operatorname{sn}^{2}\left[\omega\left(\tau-\tau_{0}\right), k\right]}{s_{31}+s_{43} \operatorname{sn}^{2}\left[\omega\left(\tau-\tau_{0}\right), k\right]} \quad \text { if } s_{4} \leq s \leq s_{3} \text {, }
$$

where

$$
\omega=\frac{\lambda_{(-)}}{2} \sqrt{s_{31} s_{42}}, \quad k^{2}=\frac{s_{43} s_{12}}{s_{13} s_{42}},
$$

where $s_{i j}=s_{j}-s_{i}$ and $\operatorname{sn}[u ; k]$ is an elliptic sine. The arbitrary constant $\tau_{0}$ is equal to zero if $s_{0}=s_{2}$ for (31) and if $s_{0}=s_{4}$ for (32). The solutions as similar the solutions (30)-(32) are obtained earlier in other variables by Cochran et al. [3].

Case $\left(F(s)=F_{4}(s), a_{0}>0\right)$. The integral (19) can be written as

$$
\lambda_{(+)} \tau=\int_{s_{3}}^{s} \frac{d s}{\sqrt{\left(s-s_{1}\right)\left(s-s_{2}\right)\left(s-s_{3}\right)\left(s-s_{4}\right)}},
$$

where $\lambda_{(+)}=\sqrt{\left(A-C_{p}\right)\left(C_{p}-B\right) /(A B)}$.

Four Real Roots. If the roots of (25) are real and only two roots correspond to the real motion

$$
s_{4}<-1<s_{3} \leq s \leq s_{2}<1<s_{1} \text {. }
$$

The variable substitution [31] in the integral (34) gives

$$
s=\frac{s_{3} s_{42}-s_{4} s_{32} \operatorname{sn}^{2}\left[\omega\left(\tau-\tau_{0}\right), k\right]}{s_{42}-s_{32} \operatorname{sn}^{2}\left[\omega\left(\tau-\tau_{0}\right), k\right]},
$$

where

$$
\omega=\frac{\lambda_{(+)}}{2} \sqrt{s_{31} s_{42}}, \quad k^{2}=\frac{s_{41} s_{32}}{s_{31} s_{42}} .
$$

The arbitrary constant $\tau_{0}$ is equal to zero if $s_{0}=s_{3}$. The solutions obtained earlier by Elipe and Lanchares [28] and the solutions (31), (32), and (36) have similar structure.

Case $\left(F(s)=F_{3}(s)\right)$. The polynomial (20) is the third-degree polynomial

$$
\begin{array}{r}
F(s)=4\left[d s-\frac{1}{2}+h\right]\left[\frac{1}{2}(1-\gamma) s^{2}-d s+\frac{b}{2}-h\right] \\
(\gamma=a, b) .
\end{array}
$$

Three Real Roots. We are only interested of the three real roots, which according to (25) and (38) can be written as

$$
s_{1}=\frac{1}{d}\left(\frac{1}{2}-h\right), \quad s_{2,3}=s_{1,2}^{\gamma}(h) .
$$

A positive value of the polynomial (38) and the condition $s \in[1,-1]$ correspond to the real motion of the gyrostat. 
TABLE 2: The exact solutions for the oblate gyrostats.

\begin{tabular}{|c|c|c|c|c|c|c|c|}
\hline Subtype & $\begin{array}{l}\text { Motion } \\
\text { type }\end{array}$ & Roots of $F_{4}(s)$ & Intervals for $s$ & $\begin{array}{l}\text { Intervals for the } \\
\text { constant } h\end{array}$ & $\begin{array}{c}\text { Add. } \\
\text { condition }\end{array}$ & $\begin{array}{l}\text { Index of the region } \\
\text { in phase space }\end{array}$ & Solution \\
\hline \multirow{5}{*}{$\mathrm{Oa}$} & Libration & $\begin{array}{c}s_{1,2}=s_{2,1}^{b} \\
s_{3,4}=s_{s} \pm i s_{k} \\
s_{s}=d /(1-a) \\
s_{k}=\sqrt{-D_{a}(h) /(1-a)}\end{array}$ & $\begin{array}{c}s_{2} \leq s \leq s_{1} \\
s_{3,4}=s_{s} \pm i s_{k}\end{array}$ & $h_{b} \geq h \geq h_{a}$ & - & $\mathrm{OaL}$ & $(30)$ \\
\hline & \multirow{4}{*}{ Rotation } & \multirow{4}{*}{$\begin{array}{l}s_{3,2}=s_{1,2}^{a}(h) \\
s_{4,1}=s_{1,2}^{b}(h)\end{array}$} & \multirow{2}{*}{$s_{2} \leq s \leq s_{1}$} & $h_{a}>h>h_{R}$ & $d>0$ & OaR1 & \multirow[t]{2}{*}{ (31) } \\
\hline & & & & $h_{a}>h>h_{L}$ & $d<0$ & OaR2 & \\
\hline & & & \multirow{2}{*}{$s_{4} \leq s \leq s_{3}$} & $h_{a}>h>h_{L}$ & $d>0$ & OaR3 & \multirow[t]{2}{*}{$(32)$} \\
\hline & & & & $h_{a}>h>h_{R}$ & $d<0$ & OaR4 & \\
\hline \multirow{5}{*}{$\mathrm{Ob}$} & & $\begin{array}{c}s_{1,2}=s_{2,1}^{b} \\
s_{3,4}=s_{s} \pm i s_{k} \\
s_{s}=d /(1-a) \\
s_{k}=\sqrt{-D_{a}(h) /(1-a)}\end{array}$ & $\begin{array}{c}s_{2} \leq s \leq s_{1} \\
s_{3,4}=s_{s} \pm i s_{k}\end{array}$ & $h_{b} \geq h \geq h_{a}$ & - & ObL1 & $(30)$ \\
\hline & Libration & $\begin{array}{l}s_{4,3}=s_{1,2}^{a}(h) \\
s_{2,1}=s_{1,2}^{b}(h)\end{array}$ & $\begin{array}{c}-1<s_{2} \leq s \leq s_{1}<1 \\
s_{4}<s_{3}<-1\end{array}$ & \multirow{2}{*}{$h_{a} \geq h>h_{L}$} & $d>0$ & ObL2 & (31) \\
\hline & & $\begin{array}{l}s_{2,1}=s_{1,2}^{a}(h) \\
s_{4,3}=s_{1,2}^{b}(h)\end{array}$ & $\begin{array}{c}-1<s_{4} \leq s \leq s_{3}<1 \\
1<s_{2}<s_{1}\end{array}$ & & $d<0$ & ObL3 & $(32)$ \\
\hline & \multirow[t]{2}{*}{ Rotation } & $\begin{array}{l}s_{4,2}=s_{1,2}^{a}(h) \\
s_{3,1}=s_{1,2}^{b}(h)\end{array}$ & $\begin{array}{c}-1<s_{2} \leq s \leq s_{1}<1 \\
s_{4}<s_{3}<-1\end{array}$ & \multirow{2}{*}{$h_{L}>h>h_{R}$} & $d>0$ & ObR1 & (31) \\
\hline & & $\begin{array}{l}s_{3,1}=s_{1,2}^{a}(h) \\
s_{4,2}=s_{1,2}^{b}(h)\end{array}$ & $\begin{array}{c}-1<s_{4} \leq s \leq s_{3}<1 \\
1<s_{2}<s_{1}\end{array}$ & & $d<0$ & ObR2 & $(32)$ \\
\hline
\end{tabular}

TABLE 3: The exact solutions for the oblate-intermediate gyrostats.

\begin{tabular}{|c|c|c|c|c|c|c|c|}
\hline Subtype & $\begin{array}{c}\text { Motion } \\
\text { type }\end{array}$ & Roots of $F_{4}(s)$ & Intervals for $s$ & $\begin{array}{l}\text { Intervals for the } \\
\text { constant } h\end{array}$ & $\begin{array}{c}\text { Add. } \\
\text { condition }\end{array}$ & $\begin{array}{l}\text { Index of the region } \\
\text { in phase space }\end{array}$ & Solution \\
\hline \multirow{3}{*}{ Oi } & \multirow[b]{2}{*}{ Libration } & $\begin{array}{c}s_{1,2}=s_{2,1}^{b}(h) \\
s_{3}=\frac{1}{d}\left(\frac{1}{2}-h\right)\end{array}$ & $s_{2} \leq s \leq s_{1}$ & \multirow[b]{2}{*}{$h_{b} \geq h>h_{L}$} & $d>0$ & OiL1 & $\begin{array}{l}(40) \\
\gamma=b\end{array}$ \\
\hline & & $\begin{array}{c}s_{1}=\frac{1}{d}\left(\frac{1}{2}-h\right) \\
s_{2,3}=s_{2,1}^{b}(h)\end{array}$ & $s_{3} \leq s \leq s_{2}$ & & $d<0$ & OiL2 & $\begin{array}{l}(41) \\
\gamma=b\end{array}$ \\
\hline & Rotation & $\begin{array}{l}s_{1,3}=s_{2,1}^{b}(h) \\
s_{2}=\frac{1}{d}\left(\frac{1}{2}-h\right)\end{array}$ & $\begin{array}{l}s_{2} \leq s \leq s_{1} \\
s_{3} \leq s \leq s_{2}\end{array}$ & $h_{L}>h>h_{R}$ & $d>0$ & OiR1 & $\begin{array}{l}(40) \\
\gamma=b \\
(41) \\
\gamma=b\end{array}$ \\
\hline
\end{tabular}

We denote the roots (39) so that the roots are located in the following sequence: $s_{1}>s_{2}>s_{3}$. The variable substitution [31] for the integral (19) gives the solutions:

$$
\begin{array}{r}
s=\frac{s_{2} s_{31}-s_{3} s_{21} \operatorname{sn}^{2}\left[\omega\left(\tau-\tau_{0}\right), k\right]}{s_{31}-s_{21} \operatorname{sn}^{2}\left[\omega\left(\tau-\tau_{0}\right), k\right]}, \\
k^{2}=\frac{s_{21}}{s_{31}} \quad\left(s_{2} \leq s \leq s_{1}\right), \\
s=s_{3}+s_{32} \operatorname{sn}^{2}\left[\omega\left(\tau-\tau_{0}\right), k\right], \\
k^{2}=\frac{s_{32}}{s_{31}} \quad\left(s_{3} \leq s \leq s_{2}\right),
\end{array}
$$

where

$$
\omega=\frac{1}{2} \sqrt{2 s_{31} a b s[d(1-\gamma)]} \quad(\gamma=a, b)
$$

The arbitrary constant $\tau_{0}$ is equal to zero if $s_{0}=s_{2}$ for (40) and if $s_{0}=s_{3}$ for (41).

The solutions (30), (31), (32), (36), (40), and (41) describe the motion of all subtypes of the gyrostats (Tables 2-6. We use the following notations in the tables:

$$
h_{\gamma}=\frac{\left[\gamma-d^{2} /(1-\gamma)\right]}{2}, \quad h_{L, R}=\frac{1}{2} \pm a b s(d) \quad(\gamma=a, b) \text {. }
$$


TABLE 4: The exact solutions for the intermediate gyrostats.

\begin{tabular}{|c|c|c|c|c|c|c|c|}
\hline Subtype & $\begin{array}{l}\text { Motion } \\
\text { type }\end{array}$ & Roots of $F_{4}(s)$ & Intervals for $s$ & $\begin{array}{l}\text { Intervals for the } \\
\text { constant } h\end{array}$ & $\begin{array}{c}\text { Add. } \\
\text { condition }\end{array}$ & $\begin{array}{l}\text { Index of the region } \\
\text { in phase space }\end{array}$ & Solution \\
\hline \multirow[b]{2}{*}{ Ia } & Libration & \multirow[b]{2}{*}{$s_{3} \leq s \leq s_{2}$} & $\begin{array}{l}s_{1,4}=s_{1,2}^{a}(h) \\
s_{3,2}=s_{1,2}^{b}(h)\end{array}$ & $h_{b} \geq h>h_{L}$ & & $\mathrm{IaL}$ & \\
\hline & Rotation & & $\begin{array}{l}s_{1,3}=s_{1,2}^{a}(h) \\
s_{4,2}=s_{1,2}^{b}(h) \\
s_{2,4}=s_{1,2}^{a}(h) \\
s_{3,1}=s_{1,2}^{b}(h)\end{array}$ & $h_{L}>h>h_{R}$ & $\begin{array}{l}d>0 \\
d<0\end{array}$ & $\begin{array}{l}\text { IaR1 } \\
\text { IaR2 }\end{array}$ & (36) \\
\hline \multirow{3}{*}{$\mathrm{Ib}$} & Libration & \multirow{3}{*}{$s_{3} \leq s \leq s_{2}$} & $\begin{array}{l}s_{1,4}=s_{1,2}^{a}(h) \\
s_{3,2}=s_{1,2}^{b}(h)\end{array}$ & $h_{b} \geq h>h_{L}$ & & IbL1 & \multirow{2}{*}{ (36) } \\
\hline & & & $\begin{array}{l}s_{2,3}=s_{1,2}^{a}(h) \\
s_{4,1}=s_{1,2}^{b}(h)\end{array}$ & $h_{R}>h \geq h_{a}$ & & IbL2 & \\
\hline & Rotation & & $\begin{array}{l}s_{1,3}=s_{1,2}^{a}(h) \\
s_{4,2}=s_{1,2}^{b}(h) \\
s_{2,4}=s_{1,2}^{a}(h) \\
s_{3,1}=s_{1,2}^{b}(h)\end{array}$ & $h_{L}>h>h_{R}$ & $\begin{array}{l}d>0 \\
d<0\end{array}$ & $\begin{array}{l}\text { IbR1 } \\
\text { IbR2 }\end{array}$ & (36) \\
\hline \multirow[b]{2}{*}{ Ic } & Libration & & $\begin{array}{l}s_{2,3}=s_{1,2}^{a}(h) \\
s_{4,1}=s_{1,2}^{b}(h)\end{array}$ & $h_{R}>h \geq h_{a}$ & & IcL & \multirow[b]{2}{*}{ (36) } \\
\hline & Rotation & $s_{3} \leq s \leq s_{2}$ & $\begin{array}{l}s_{1,3}=s_{1,2}^{a}(h) \\
s_{4,2}=s_{1,2}^{b}(h) \\
s_{2,4}=s_{1,2}^{a}(h) \\
s_{3,1}=s_{1,2}^{b}(h)\end{array}$ & $h_{L}>h>h_{R}$ & $\begin{array}{l}d>0 \\
d<0\end{array}$ & $\begin{array}{l}\text { IcR1 } \\
\text { IcR2 }\end{array}$ & \\
\hline
\end{tabular}

TABLE 5: The exact solutions for the prolate-intermediate gyrostats.

\begin{tabular}{|c|c|c|c|c|c|c|c|}
\hline Subtype & Motion type & Roots of $F_{4}(s)$ & Intervals for $s$ & $\begin{array}{c}\text { Intervals for the } \\
\text { constant } h\end{array}$ & $\begin{array}{c}\text { Add. } \\
\text { condition }\end{array}$ & $\begin{array}{l}\text { Index of the region } \\
\text { in phase space }\end{array}$ & Solution \\
\hline \multirow{3}{*}{$\mathrm{Pi}$} & & $\begin{array}{l}s_{1}=\frac{1}{d}\left(\frac{1}{2}-h\right) \\
s_{2,3}=s_{1,2}^{a}(h)\end{array}$ & $s_{3} \leq s \leq s_{2}$ & \multirow[b]{2}{*}{$h_{R}>h \geq h_{a}$} & $d>0$ & PiL1 & $\begin{array}{l}(41) \\
\gamma=a\end{array}$ \\
\hline & Libration & $\begin{array}{l}s_{1,2}=s_{1,2}^{a}(h) \\
s_{3}=\frac{1}{d}\left(\frac{1}{2}-h\right)\end{array}$ & $s_{2} \leq s \leq s_{1}$ & & $d<0$ & PiL2 & $\begin{array}{l}(40) \\
\gamma=a\end{array}$ \\
\hline & Rotation & $\begin{array}{l}s_{1,3}=s_{1,2}^{a}(h) \\
s_{2}=\frac{1}{d}\left(\frac{1}{2}-h\right)\end{array}$ & $\begin{array}{l}s_{3} \leq s \leq s_{2} \\
s_{2} \leq s \leq s_{1}\end{array}$ & $h_{L}>h>h_{R}$ & $d>0$ & PiR1 & $\begin{array}{l}(41) \\
\gamma=a \\
(40) \\
\gamma=a\end{array}$ \\
\hline
\end{tabular}

\section{A Complete Set of the Phase Trajectories and the Exact Solutions}

In this section the phase portraits are shown in the case $d>0$ only. Keeping in mind that at $d<0$ the stationary points are obtained as a mirror image relative to abscissa axis according to the solutions (11)-(14), we use the following values of the gyrostat's parameters for the numerical calculations

$$
A=0.85 \mathrm{~kg} \times \mathrm{m}^{2}, \quad B=0.65 \mathrm{~kg} \times \mathrm{m}^{2}, \quad d=0.05 c^{-1} .
$$

5.1. Phase Space Structure for the Oblate Gyrostats. The solutions for the oblate gyrostats are given in Table 2. Figure 4 illustrates the case when the saddles are on verticals $l_{s}= \pm \pi / 2$ $\left(\left|s_{s}\right|<1\right)$. The saddles can reach the horizontal $s_{s}=1$ as shown in Figure 5.

5.2. Phase Space Structure for the Oblate-Intermediate Gyrostats. Table 3 shows the solutions for the oblate-intermediate gyrostats $(a=1)$. The corresponding phase structure is shown on Figure 6.

5.3. Phase Space Structure for the Intermediate Gyrostats. Other solutions for this type of the gyrostats are presented in Table 4 and the phase structures are shown in Figures 68. The phase structure for the Subtype Ia is similar to the 
TABLE 6: The exact solutions for the prolate gyrostats.

\begin{tabular}{|c|c|c|c|c|c|c|c|}
\hline Subtype & $\begin{array}{l}\text { Motion } \\
\text { type }\end{array}$ & Roots of $F_{4}(s)$ & Intervals for $s$ & $\begin{array}{l}\text { Intervals for the } \\
\text { constant } h\end{array}$ & $\begin{array}{l}\text { Add. } \\
\text { condition }\end{array}$ & $\begin{array}{l}\text { Index of the region } \\
\text { in phase space }\end{array}$ & Solution \\
\hline \multirow{5}{*}{$\mathrm{Pa}$} & \multirow[b]{3}{*}{ Libration } & $\begin{array}{l}s_{1,2}=s_{1,2}^{b}(h) \\
s_{3,4}=s_{1,2}^{a}(h)\end{array}$ & $\begin{array}{l}-1<s_{4} \leq s \leq s_{3}<1 \\
1<s_{2}<s_{1}\end{array}$ & \multirow{2}{*}{$h_{R}>h \geq h_{b}$} & $d>0$ & PaL1 & (32) \\
\hline & & $\begin{array}{l}s_{1,2}=s_{1,2}^{a}(h) \\
s_{3,4}=s_{1,2}^{b}(h)\end{array}$ & $\begin{array}{l}-1<s_{2} \leq s \leq s_{1}<1 \\
s_{4}<s_{3}<-1\end{array}$ & & $d<0$ & $\mathrm{PaL} 2$ & $(31)$ \\
\hline & & $\begin{array}{l}s_{1,2}=s_{1,2}^{a} \\
s_{3,4}=s_{s} \pm i s_{k} \\
s_{s}=d /(1-b) \\
s_{k}=\sqrt{-D_{b}(h)} /(1-b)\end{array}$ & $\begin{array}{l}s_{2} \leq s \leq s_{1} \\
s_{3,4}=s_{s} \pm i s_{k}\end{array}$ & $h_{b} \geq h \geq h_{a}$ & $d \in \mathbb{R}$ & PaL3 & $(30)$ \\
\hline & \multirow[b]{2}{*}{ Rotation } & $\begin{array}{l}s_{2,4}=s_{1,2}^{a}(h) \\
s_{1,3}=s_{1,2}^{b}(h)\end{array}$ & $\begin{array}{l}-1<s_{4} \leq s \leq s_{3}<1 \\
1<s_{2}<s_{1}\end{array}$ & \multirow{2}{*}{$h_{L}>h>h_{R}$} & $d>0$ & PaR1 & (32) \\
\hline & & $\begin{array}{l}s_{2,4}=s_{1,2}^{b}(h) \\
s_{1,3}=s_{1,2}^{a}(h)\end{array}$ & $\begin{array}{l}-1<s_{2} \leq s \leq s_{1}<1 \\
s_{4}<s_{3}<-1\end{array}$ & & $d<0$ & $\mathrm{PaR} 2$ & $(31)$ \\
\hline \multirow{5}{*}{$\mathrm{Pb}$} & Libration & $\begin{array}{l}s_{1,2}=s_{1,2}^{a} \\
s_{3,4}=s_{s} \pm i s_{k} \\
s_{s}=d /(1-b) \\
s_{k}=\sqrt{-D_{b}(h)} /(1-b)\end{array}$ & $\begin{array}{l}s_{2} \leq s \leq s_{1} \\
s_{3,4}=s_{s} \pm i s_{k}\end{array}$ & $h_{b} \geq h \geq h_{a}$ & $d \in \mathbb{R}$ & $\mathrm{PbL}$ & $(30)$ \\
\hline & \multirow{4}{*}{ Rotation } & $s_{3,2}=s_{1,2}^{a}(h)$ & \multirow{2}{*}{$s_{2} \leq s \leq s_{1}$} & $h_{R}>h>h_{b}$ & $d>0$ & $\mathrm{PbR} 1$ & \multirow{2}{*}{ (31) } \\
\hline & & $s_{4,1}=s_{1,2}^{b}(h)$ & & $h_{L}>h>h_{b}$ & $d<0$ & $\mathrm{PbR} 2$ & \\
\hline & & $s_{1,4}=s_{1,2}^{a}(h)$ & \multirow{2}{*}{$s_{4} \leq s \leq s_{3}$} & $h_{L}>h>h_{b}$ & $d>0$ & $\mathrm{PbR} 3$ & \multirow[t]{2}{*}{$(32)$} \\
\hline & & $s_{2,3}=s_{1,2}^{b}(h)$ & & $h_{R}>h>h_{b}$ & $d<0$ & $\mathrm{PbR} 4$ & \\
\hline
\end{tabular}

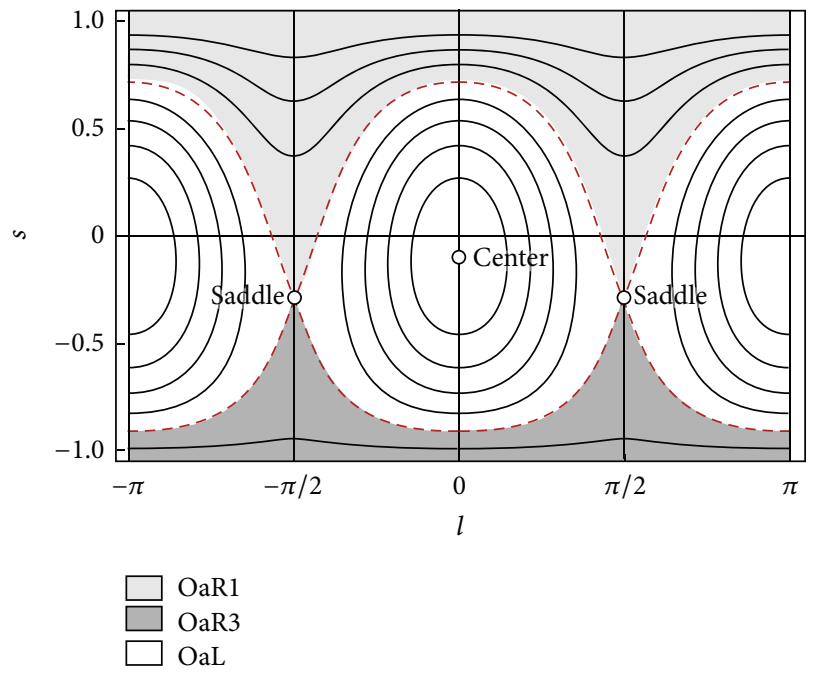

FIGURE 4: Phase space structure for the oblate gyrostat, indexes of the region in phase space: OaR1, OaR3, and OaL $(a=1.17647, b=$ $\left.1.53846, l_{c}=0, s_{c}=-0.093, l_{s}=\pi / 2, s_{s}=-0.283\right)$. Here and further the separatrices are denoted by a dashed line.

phase portrait in Figure 6. We see a doubling of centers and saddles ("old" and "new") for the Subtype Ib (Figure 7). The "old" centers and "old" saddles disappear for the Subtype Ic (Figure 8).

5.4. Phase Space Structure for the Prolate-Intermediate Gyrostats. The exact solutions for the prolate-intermediate

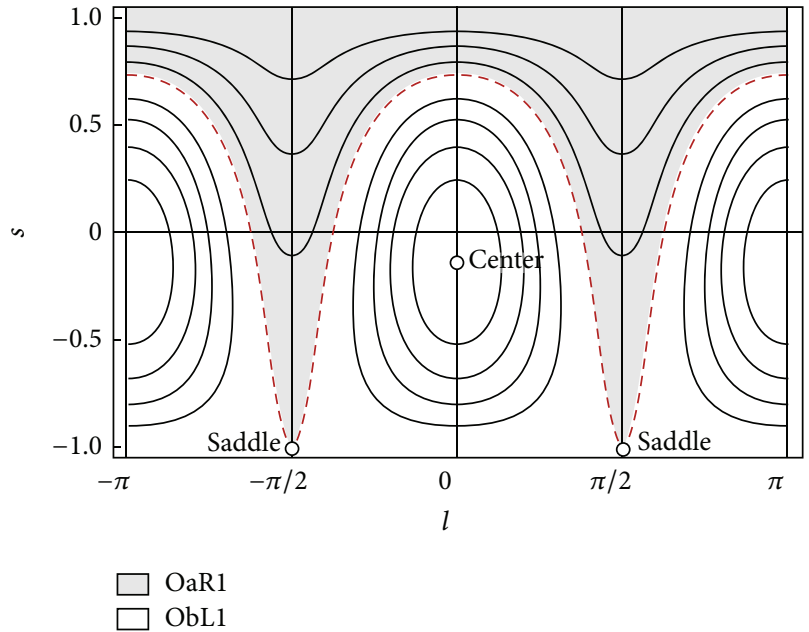

FIGURE 5: Phase space structure for the oblate gyrostat, indexes of the region in phase space: OaR1, ObL1, and OaL $(a=1.05, b=$ $\left.1.3731, l_{c}=0, s_{c}=-0.134, l_{s}=\pi / 2, s_{s}=-1\right)$.

gyrostats are presented in Table 5. The motions of the gyrostats for the type $\mathrm{Pi}$ are qualitatively similar to the motions of the Subtype Ic (Figure 8).

5.5. Phase Space Structure for the Prolate Gyrostats. Table 6 shows the solutions for the prolate gyrostats. We can see the similarity between the Subtype Ic the the Subtypes $\mathrm{Pa}$ (Figure 8). In case of the Subtype $\mathrm{Pb}$ we observe a similarity 


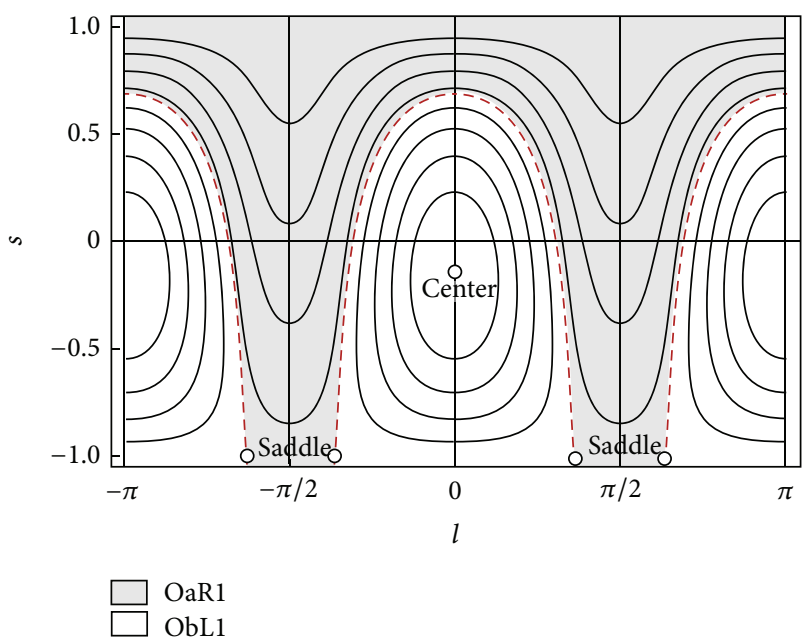

FIGURE 6: Phase space structure for the oblate-intermediate gyrostat, indexes of the region in phase space: ObR1, ObL1 $(a=1, b=1.3077$, $\left.l_{c}=0, s_{c}=-0.1625, l_{s}= \pm 1.15588, s_{s}=-1\right)$.

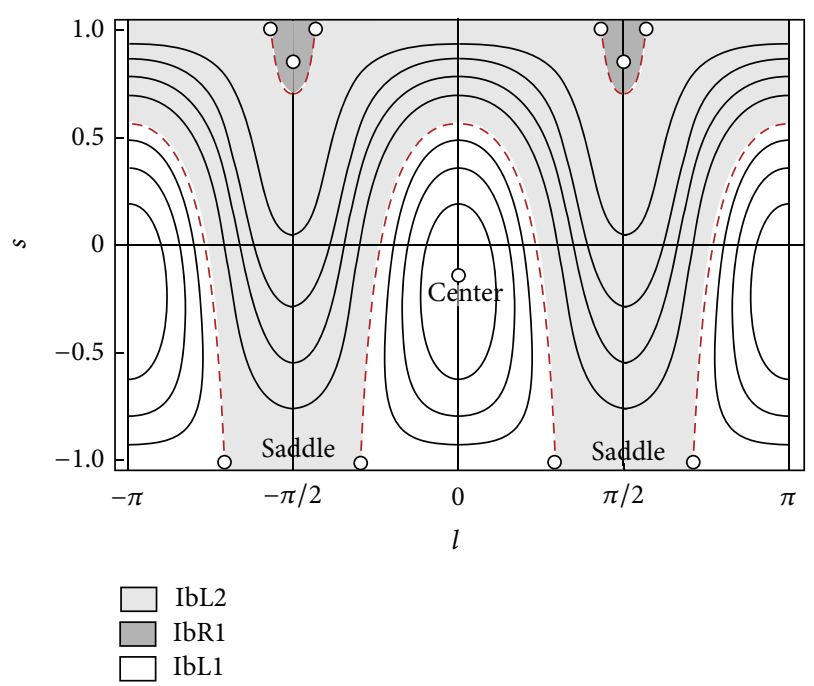

FIGURE 7: Phase space structure for the intermediate gyrostat, indexes of the region in phase space $C_{P}=0.8 \mathrm{~kg} \cdot \mathrm{m}^{2} \Rightarrow a=$ $0.94118, b=1.23077\left(l_{c}=0, s_{c}=-0.21667, l_{c}= \pm \pi / 2, s_{c}=0.85\right.$, $\left.l_{s}= \pm 0.910932, s_{s}=-1, l_{s}= \pm 1.39534, s_{s}=1\right)$.

with the Subtype Oa (Figure 4), when the phase portrait is shifted along the horizontal axis at $\pm \pi / 2$ (Figure 9).

Thus we have observed the evolution of the phase space, depending on the parameters of the gyrostat.

\section{Conclusion}

This paper is a development of the results obtained in Aslanov [24, 27]. Two new gyrostats types are added (oblateintermediate and prolate-intermediate) and the exact solutions are found, including three the new solutions. The results

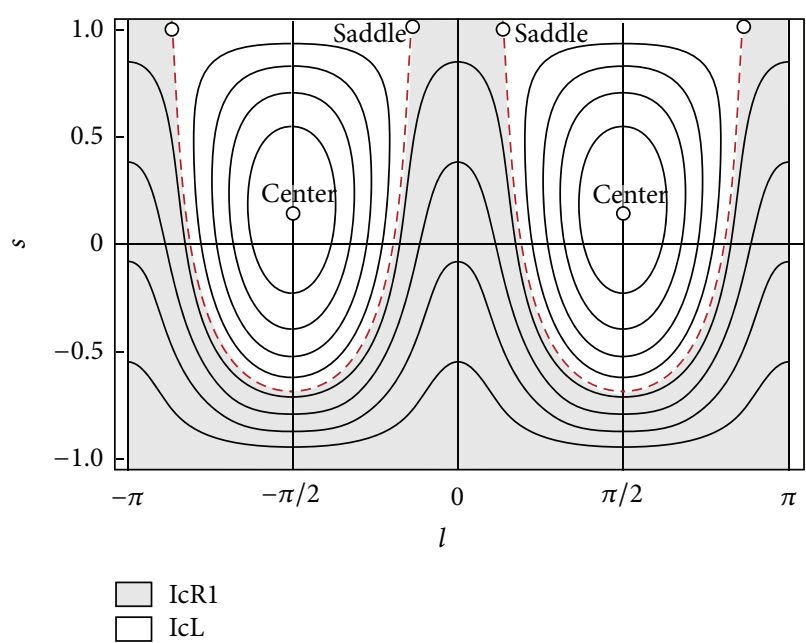

Figure 8: Phase space structure for the intermediate gyrostat, indexes of the region in phase space $C_{P}=0.6825 \mathrm{~kg} \cdot \mathrm{m}^{2} \Rightarrow a=$ $0.80294, b=1.05$. $\left(l_{c}= \pm \pi / 2, s_{c}=0.253731, l_{s}= \pm 0.689575, s_{s}=1\right)$.

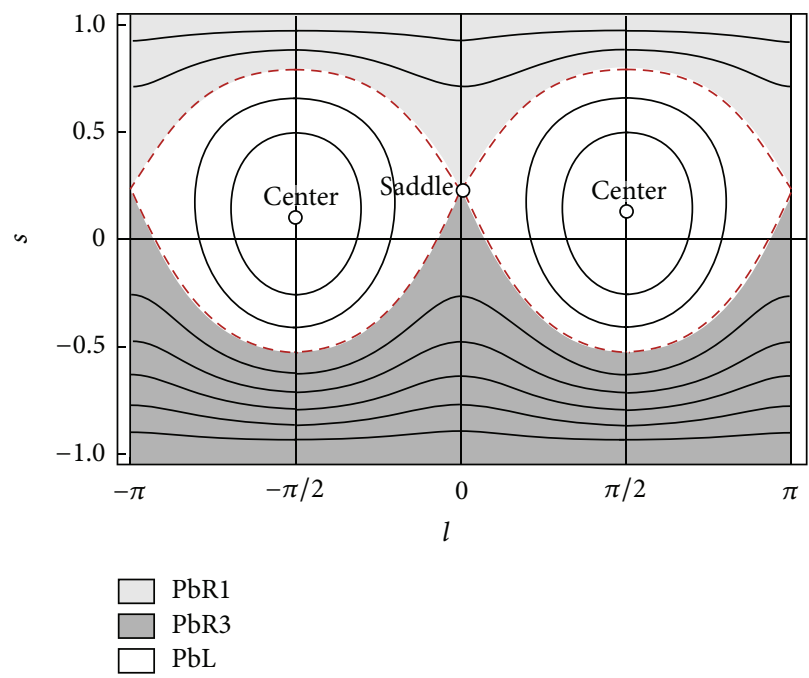

FIgURE 9: Phase space structure of the prolate gyrostat, indexes of the region in phase space: $C_{P}=0.5 \mathrm{~kg} \cdot \mathrm{m}^{2} \Rightarrow a=0.58823, b=$ $0.76923\left(l_{c}= \pm \pi / 2, s_{c}=0.1214, l_{s}=0, s_{s}=0.21667\right)$.

of the study can be useful for the analysis of dual-spin spacecraft dynamics.

\section{Conflict of Interests}

The author declares that there is no conflict of interests regarding the publication of this paper.

\section{Acknowledgments}

The author is grateful to Dr. Radwa Ibrahim for the invitation to publish in this journal. This work was supported by Ministry of education and science of Russia (Contract no. 9.540.2014/K). 


\section{References}

[1] E. Leimanis, "Heavy rigid body," in The General Problem of the Motion of Coupled Rigid Bodies about a Fixed Point, vol. 7 of Springer Tracts in Natural Philosophy, pp. 1-136, Springer, Berlin, Germany, 1965.

[2] T. R. Kane, "Solution of the equations of rotational motion for a class of torque-free gyrostats," AIAA Journal, vol. 8, no. 6, pp. 1141-1143, 1970.

[3] J. E. Cochran, P. H. Shu, and S. D. Rew, "Attitude motion of asymmetric dual-spin spacecraft," Journal of Guidance, Control, and Dynamics, vol. 5, no. 1, pp. 37-42, 1982.

[4] C. D. Hall, "Equivalence of two classes of dual-spin spacecraft spinup problems," Journal of Guidance, Control, and Dynamics, vol. 15, no. 4, pp. 1032-1034, 1992.

[5] C. D. Hall, "Spinup dynamics of gyrostats," Journal of Guidance, Control, and Dynamics, vol. 18, no. 5, pp. 1177-1183, 1995.

[6] C. D. Hall, "Escape from gyrostat trap states," Journal of Guidance, Control, and Dynamics, vol. 21, no. 3, pp. 421-426, 1998.

[7] C. D. Hall and R. H. Rand, "Spinup dynamics of axial dual-spin spacecraft," Journal of Guidance, Control, and Dynamics, vol. 17, no. 1, pp. 30-37, 1994.

[8] A. Elipe, "Gyrostats in free rotation," International Astronomical Union Colloquium, vol. 165, pp. 1-8, 1991.

[9] A. Elipe, "Hamiltonianos cuadráticos en la esfera unidad o Giróstato en movimiento libre: dos problemas equivalentes," Revista de la Academia de Ciencias Exactas, vol. 53, pp. 5-28, 1998 (Spanish).

[10] A. Elipe and V. Lanchares, "Phase flow of an axially symmetrical gyrostat with one constant rotor," Journal of Mathematical Physics, vol. 38, no. 7, pp. 3533-3544, 1997.

[11] A. Elipe and V. Lanchares, "Two equivalent problems: gyrostats in free motion and parametric quadratic Hamiltonians," Mechanics Research Communications, vol. 24, no. 6, pp. 583590, 1997.

[12] A. Elipe, M. Arribas, and A. Riaguas, "Complete analysis of bifurcations in the axial gyrostat problem," Journal of Physics A: Mathematical and General, vol. 30, no. 2, pp. 587-601, 1997.

[13] V. Lanchares, M. Iñarrea, and J. P. Salas, "Spin rotor stabilization of a dual-spin spacecraft with time dependent moments of inertia," International Journal of Bifurcation and Chaos in Applied Sciences and Engineering, vol. 8, no. 3, pp. 609-617, 1998.

[14] M. Iñarrea and V. Lanchares, "Chaos in the reorientation process of a dual-spin spacecraft with time-dependent moments of inertia," International Journal of Bifurcation and Chaos in Applied Sciences and Engineering, vol. 10, no. 5, pp. 997-1018, 2000.

[15] P. C. Hughes, Spacecraft Attitude Dynamics, John Wiley \& Sons, New York, NY, USA, 1986.

[16] J. Koiller, "A mechanical system with a 'wild" horseshoe," Journal of Mathematical Physics, vol. 25, no. 5, pp. 1599-1604, 1984.

[17] J. A. Serret, "Mémoire sur l'emploi de la méthode de la variation des arbitraires dans la théorie des mouvements de rotation," Mémoires de l'Academie des Sciences de Paris, vol. 55, pp. 585616, 1866

[18] H. Andoyer, Cours de Mécanique Céléste, Gauthier-Villars, Paris, France, 1923.

[19] A. Deprit, "Free rotation of a rigid body studied in the phase plane," The American Journal of Physics, vol. 35, pp. 424-428, 1967.
[20] A. Deprit and A. Elipe, "Complete reduction of the EulerPoinsot problem," The American Astronautical Society, vol. 41, no. 4, pp. 603-628, 1993.

[21] X. Tong, B. Tabarrok, and F. P. Rimrott, "Chaotic motion of an asymmetric gyrostat in the gravitational field," International Journal of Non-Linear Mechanics, vol. 30, no. 3, pp. 191-203, 1995.

[22] V. S. Aslanov and A. V. Doroshin, "Chaotic dynamics of an unbalanced gyrostat," Journal of Applied Mathematics and Mechanics, vol. 74, no. 5, pp. 524-535, 2010.

[23] V. S. Aslanov, "Dynamics of free dual-spin spacecraft," Engineering Letters, vol. 19, pp. 271-278, 2011.

[24] V. S. Aslanov, "Integrable cases in the dynamics of axial gyrostats and adiabatic invariants," Nonlinear Dynamics, vol. 68, no. 1-2, pp. 259-273, 2012.

[25] V. Aslanov and V. Yudintsev, "Dynamics and chaos control of gyrostat satellite," Chaos, Solitons and Fractals, vol. 45, no. 9-10, pp. 1100-1107, 2012.

[26] V. S. Aslanov and V. V. Yudintsev, "Dynamics and control of dual-spin gyrostat spacecraft with changing structure," Celestial Mechanics \& Dynamical Astronomy, vol. 115, no. 1, pp. 91-105, 2013.

[27] V. S. Aslanov, "A note on the "exact solutions for angular motion of coaxial bodies and attitude dynamics of gyrostat-satellites'”, International Journal of Non-Linear Mechanics, vol. 58, pp. 305306, 2014.

[28] A. Elipe and V. Lanchares, "Exact solution of a triaxial gyrostat with one rotor," Celestial Mechanics \& Dynamical Astronomy, vol. 101, no. 1-2, pp. 49-68, 2008.

[29] Y. A. Sadov, "The action-angle variables in the Euler-Poinsot problem," Preprint No. 22, Akademii Nauk SSSR, 1970.

[30] A. Elipe, "On the attitude dynamics of perturbed triaxial rigid bodies," Universidad de Zaragoza, 2002.

[31] G. Korn and T. Korn, Mathematical Handbook, McGraw-Hill, New York, NY, USA, 1968. 


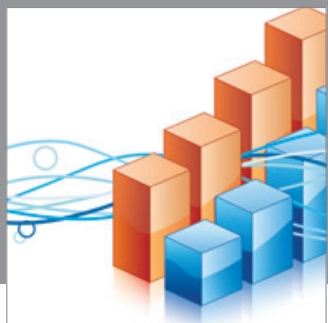

Advances in

Operations Research

mansans

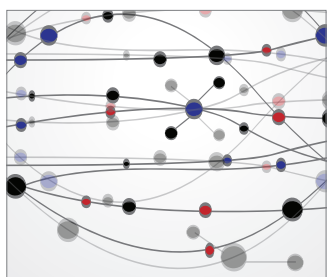

The Scientific World Journal
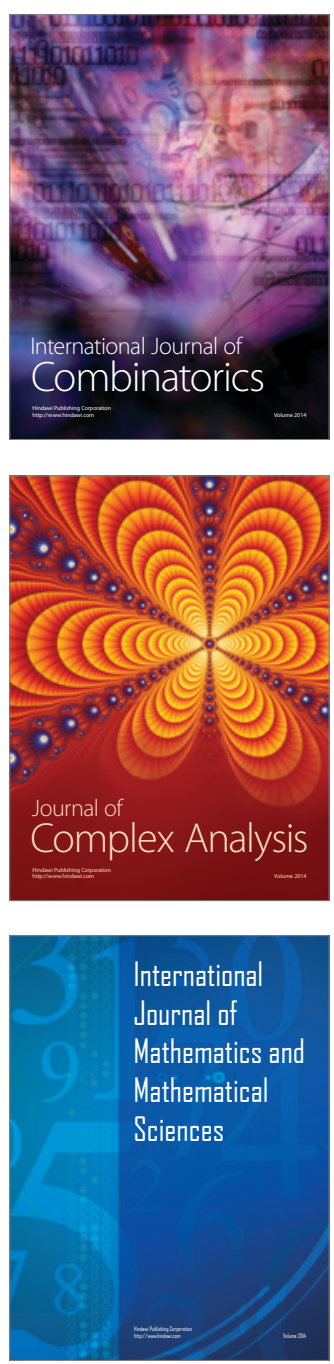
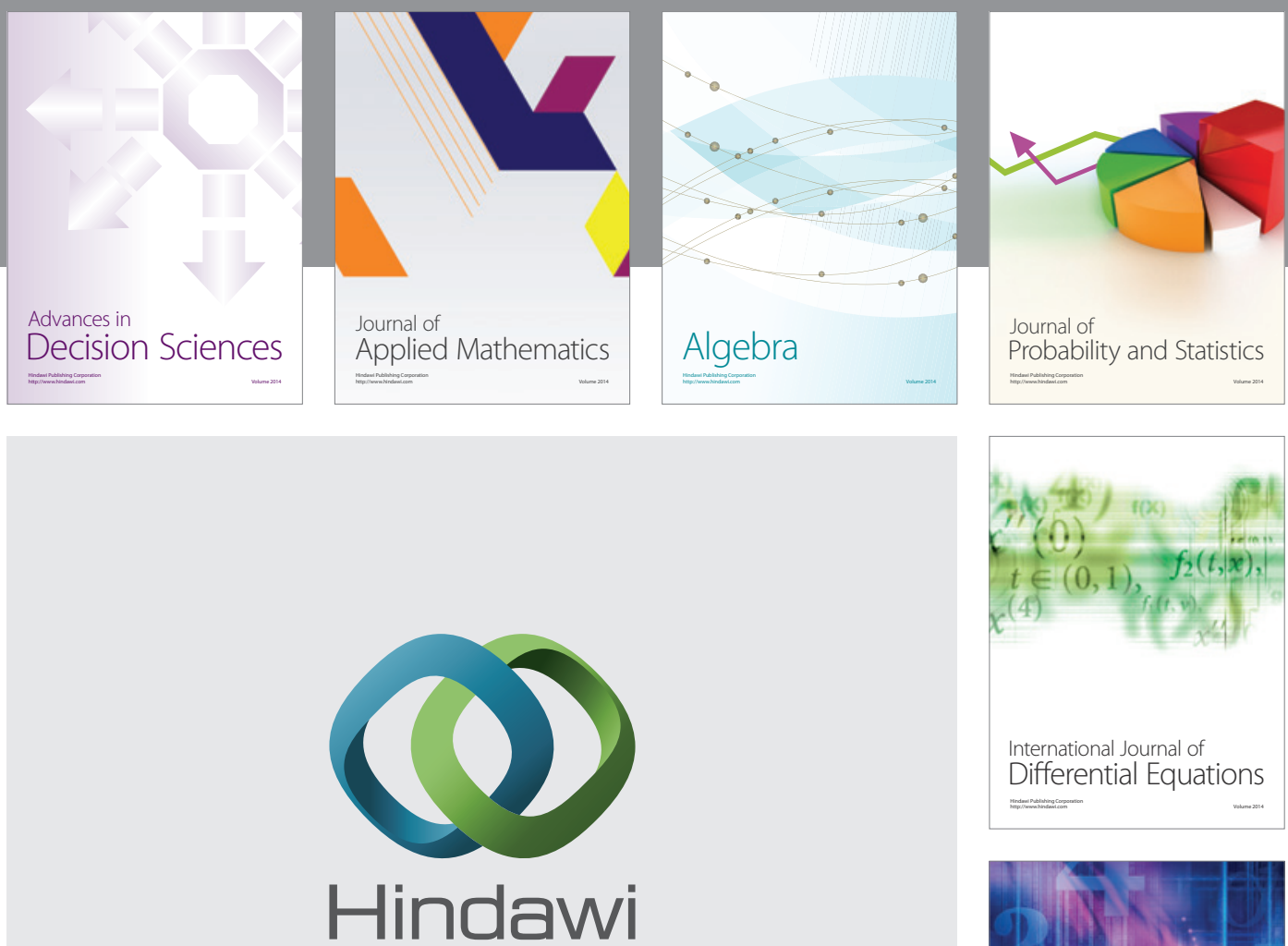

Submit your manuscripts at http://www.hindawi.com
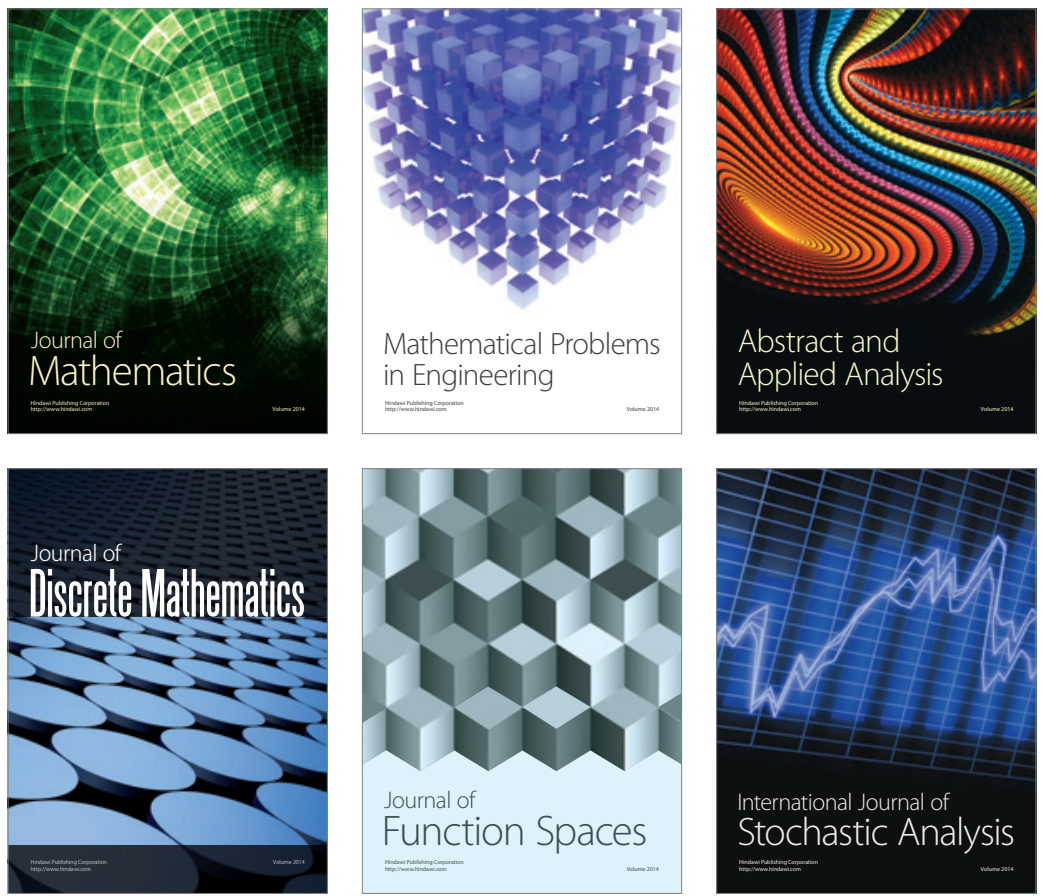

Journal of

Function Spaces

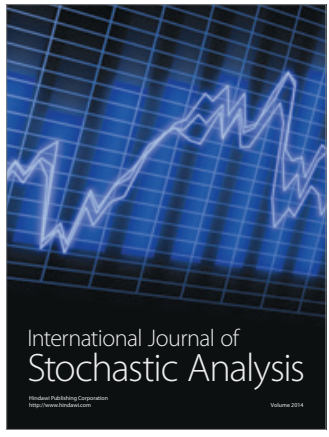

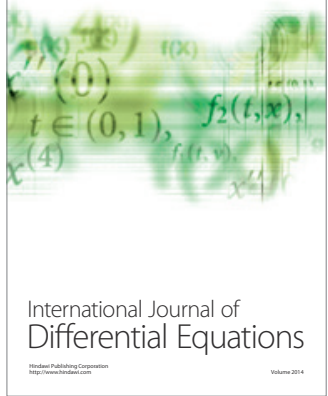
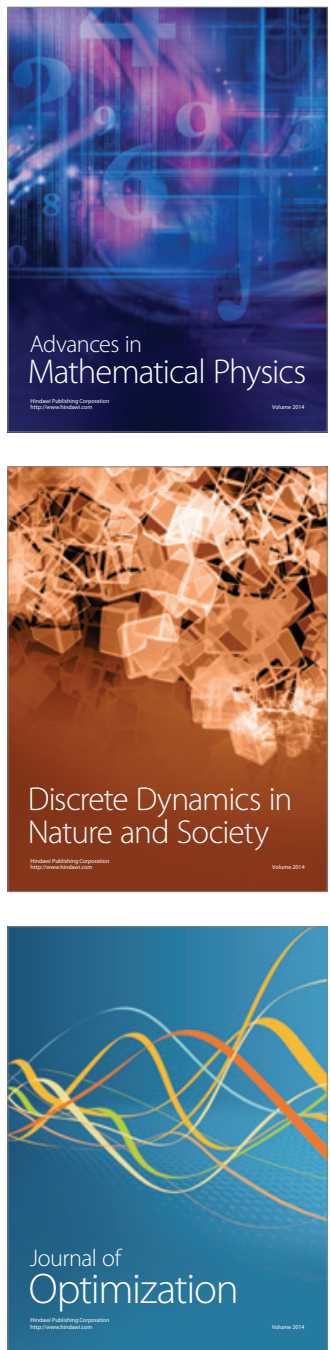